\title{
Investigation and Study on the Status of the College Students with Left-Behind Experience in China
}

\author{
Zhang Yong a,b,* ${ }^{\text {, Jiang Wulin }}{ }^{\mathrm{a}}$, Xiang Yunbo ${ }^{\mathrm{a}, \mathrm{b}}$ \\ ${ }^{a}$ School of Architecture and Urban Planning of Hunan University of Science and Technology, Xiangtan, 41120, \\ China \\ ${ }^{b}$ Regional geography of Hunan University of Science and Technology, Xiangtan, 41120, China
}

\begin{abstract}
College students with left-behind experience are a special group and have different characteristics compared to students without left-behind experience. Based on investigation, college students with left-behind experience and interview some students around at them, this paper analyzed the basic situations of the college students with left-behind experience, as well as the key factors leading to these situations. Finally, the corresponding suggestions and expectations to improve the situations are put forward.
\end{abstract}

Index Terms: Left-behind children; College students; Left-behind experience.

(C) 2014 Published by MECS Publisher. Selection and/or peer review under responsibility of the Research Association of Modern Education and Computer Science.

\section{Introduction}

Since the Chinese reform, with the rapid development of urbanization and the economic acceleration, a large number of surplus labours began to emerge in the rural of China and gradually enter the coastal economically developed area. So the world-famous wave of migrant peasant laborers arose in China. However, many of them have become parents, as a result, a special group, the left-behind children, appeared in rural areas due to being uprooted from their homes.

Obviously, farmers working in cities not only effectively solved the problem of rural surplus labor, but also relieved the employment pressure in rural areas, as well as provided adequate and cheap labors for the city development. And it also promoted economic development and construction of the cities $[1,2,3]$. But at the same time, it also produced many adverse effects, one of the most noticeable special social problems are "leftbehind child". Actually, the family is the first classroom for children's education and parental education plays an irreplaceable role in childhood and the later growth process. For left-behind children in significant psychological and physiological development stage, a series of outstanding problems, such as life, education,

* Corresponding author. Tel.: +86 15173265757

E-mail address:292278@qq.com 
emotional and psychological, etc., will be triggered due to the lack of parental control. At present, China's floating population has surpassed billions every year, among these, the number of children and adolescents who go along with their parents at the stage of compulsory education is about 3000000 . However, left-behind children are far beyond the above number [4]. As everyone knows, college students with certain knowledge accomplishment and practical experience are the mainstay of the motherland's future. However, because of the special left-behind experience, inevitably, the college students with left-behind experience differ from the college students without left-behind experience in the aspects of life, psychology, emotion and personality. Paying close attention to the students' status is not just a discussion on the left-behind children's training mode, but also an effective method is deeper into understanding them [5, 6, 7]. And it is of great advantage to solve the problems of left-behind child.

\section{Materials and Methods}

The students of Hunan University of Science and Technology are chosen as the research objects in this paper. Hunan University of Science and Technology is situated in Xiangtan City for the National Admissions. So its students come from all parts of the country and have a large number of students. At the same time, the college is just in the juncture of middle and west of China. Consequently, it has very good representation.

The research used questionnaire and interview methods to study. 205 questionnaires were issued randomly for college students with left-behind experience; 200 effective questionnaires were recalled, and the recovery was $97.6 \%$. At the same time, interview with face-to-face was carried out in college students who are familiar with the college students with left-behind experience and had not the left-behind experience randomly.

\section{Basic status of the college students with left-behind experience}

\subsection{Economic conditions}

\subsubsection{Living expenses}

The direct cause of "left-behind children" problem is the backward rural economy. For the pursuit of higher economic income, farmers have to go to the city to look for other job opportunities to improve the economic situation of the family. Investigation shows the monthly living expenses of the college students with left-behind experience below $500 ¥$ are the most, accounting for 50.5\%; followed by the 500-1000 $¥$, a percentage of $38.5 \%$; 1000-1500 $¥$ was $6 \%$; while more than $1500 ¥$ is only $5 \%$ (Fig. 1). Thus, most of their living expenses are not much. Except for living expenses, other expenses had to be reduced further to cope with their live.

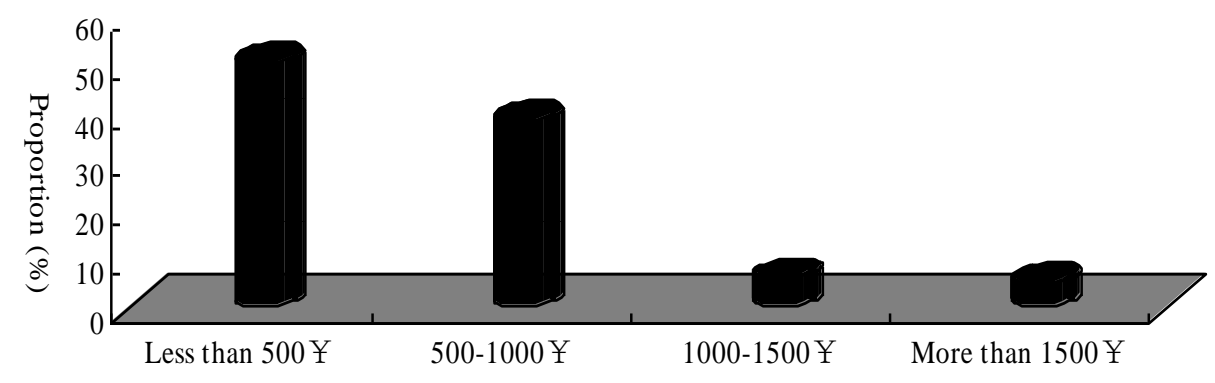

Fig. 1. Monthly living expenses

\subsubsection{Family incomes}


For family monthly income of the college students with left-behind experience, there is a very considerable difference (Fig. 2). Most levels are 1000-3000¥, accounted for 63.3\%; the second level is less than $1000 ¥$, accounted for $18.7 \%$; the third level is $3000-5000 ¥$, accounted for $11.2 \%$; the minimum is more than $5000 ¥$, only accounted for $6.6 \%$. As it can be viewed, most of the family incomes are general, and there is quite a lot of monthly family income of less than $1000 ¥$. Obviously, it’ s extremely difficult to train a college student only relying on the monthly income of less than $1000 ¥$. If attending expensive professional, it may not collect the tuition fee a year.

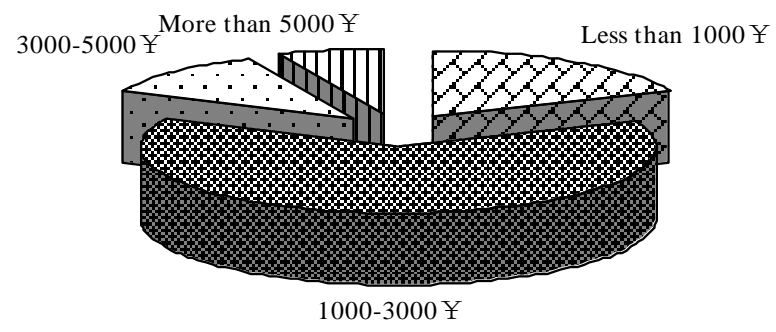

Fig. 2. Family monthly income of the college students with left-behind experience

\subsection{Learning situations}

\subsubsection{Learning achievement}

For left-behind children, because their parents so often go out that the parents neglected the children's living and learning control and were short of supervision and guidance of the children's learning, the children's study problems became progressively worse. Depending on statistics, the learning ability of left-behind children is poor and the learning achievements of the last ten students are that of left-behind child.

According to table 1, a very good learning achievement of the college students with left-behind experience was only 8.2 percent; a better achievement accounted for $43.8 \%$; the average achievement accounted for $33.5 \%$; a poor achievement accounted for $11.4 \%$, and a very poor achievement accounted for $3.1 \%$. Obviously, this result showed that the learning achievements of the college students with left-behind experience are not really ideal.

Table 1. Status of the learning achievement of the college students with left-behind experience

\begin{tabular}{lllllc}
\hline Learning achievement & Very good & Better & Average & Poor & Very poor \\
\hline Proportion & $8.2 \%$ & $43.8 \%$ & $33.5 \%$ & $11.4 \%$ & $3.1 \%$ \\
\hline
\end{tabular}

\subsubsection{Learning attitude}

The survey found the college students with left-behind experience without playing truant and listen attentively were only a few. And most of them rarely played truant and are not attentive listening to the class occasionally. But there are still a few people without attending the classes, even rarely going to class (Table 2). The survey results showed that the learning attitude of most college students with left-behind experience is not serious, even a lessee faire attitude. And the survey also analyzed the academic achievements of students with left-behind experience after their parents went away. The statistical results show that only $18.4 \%$ of students' academic achievements become very good. However, the academic achievements of $48.7 \%$ college students 
with left-behind experience are better; the average academic achievements account for $21.3 \%$, and the poor and very poor academic achievements account for $10.9 \%$ and $0.4 \%$ respectively. Obviously, because parents go out, some bad phenomena, such as bad learning attitude, not strong learning initiative and lack of interest, and so on, also influence the college students with left-behind experience.

Table 2 Attending classes of college students with left-behind experience

\begin{tabular}{lllc}
\hline \multicolumn{2}{c}{ Enthusiasm of attending class } & \multicolumn{2}{c}{ Attitude of attending class } \\
Frequency of truancy & Proportion $(\%)$ & Seriously studying degree & Proportion $(\%)$ \\
\hline None & 10.3 & Very serious & $10.8 \%$ \\
Fewer & 54.0 & Unseriousness occasionally & $60.5 \%$ \\
Frequently & 33.3 & Serious frequently & $27.5 \%$ \\
Seldom & 2.4 & Serious nearly & $1.2 \%$ \\
\hline
\end{tabular}

\subsection{Interpersonal situations}

Juvenile period is a critical period of personality formation and development. During this period, parental care, good family environment and education play irreplaceable roles for the healthy development of youthful character. Owing to the lack of perfect love and communication, left-behind children will produce psychological barriers of communication with people easily. They often have problems of poor communication, not emotional communication with other people and poor ability of interpersonal communication $[8,9,10,11]$.

The interpersonal situation of college students with left-behind experience is not particularly optimistic. Very few of them take an active part in social activities, only accounted for $4.7 \%$. The social activities' enthusiasm of the vast majority of college students with left-behind experience is very general, in addition, $3.3 \%$ students are not active. Some of them even said that they didn't want to take part in social activities, if they didn't have to go. Some phenomenon (e.g. asocial, hating activities in places with lots of people and tension as meeting many people) is particularly prominent (Fig. 3).

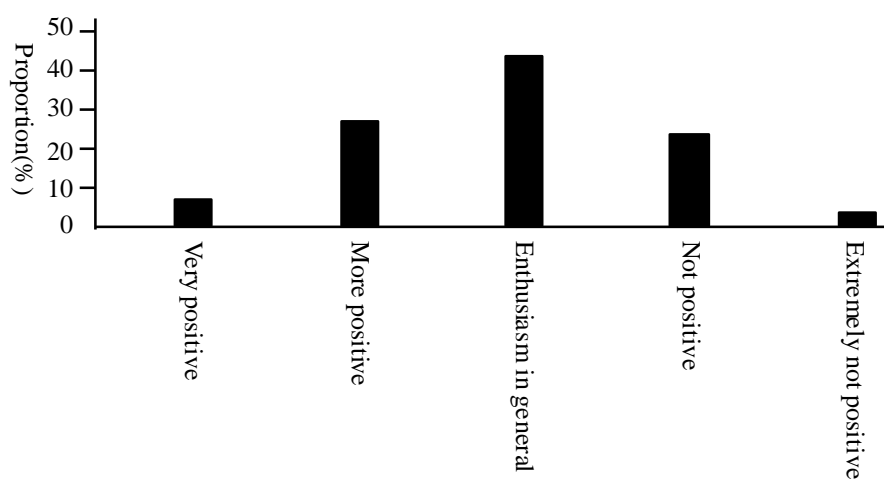

Fig. 3. Enthusiasm to participate in social activities

In addition, only $8.1 \%$ of college students with left-behind experience think they have a lot of friends and $37.3 \%$ and $38.8 \%$ feel they have pretty much and many friends respectively. However, $11.5 \%$ think their friends are not much, another $4.3 \%$ feel they have very few friends. The above data not only shows that most college students with left-behind experience do not have a strong ability of communication, but also a lot of them can't communicate with people. This reflects the defense and resistance of these students against people around them is very strong. For the college students with left-behind experience, all performance, such as 
exclusion of others, not easy to communicate with others, not to take the initiative to care for others and even not to complete a normal conversation perfectly are specific and severe performance of bad communication abilities.

\subsection{Personality characteristics}

\subsubsection{Personality tendency}

For left-behind children, introversion, solitary, inferiority and irascibility have always been considered the main character flaws. However, some of these symptoms which retained in the college students with leftbehind experience are not clear. In all the copies of the questionnaire, the proportion which they think themselves introverted is $29.8 \%$. On the contrary, the number of "outgoing and lively" is $29.9 \%$. And the remaining $31.3 \%$ of people are not sure about their personality type or belong to dual character. Thus, our survey shows the difference of the character tendency between the college students with left-behind experience and the college students without left-behind experience is not large. And unlike some other reports said the majority of left-behind children were introverted and withdrawn.

\subsubsection{Level of confidence}

Fig. 4 showed that the most of the college students with left-behind experience were confident, even $14.5 \%$ are very confident. But a particular worry is that $19 \%$ of them feel inferior and $17 \%$ think they are not confident, always felt inferior to men and abandon yourself to despair.

For left-behind children, because their parents are not around, most children were farmed out to relatives. Therefore, left-behind children are lack of security and a sense of belonging so that many people are prone to produce adverse psychological of inferiority. Although the college students with left-behind experience have already been away from the left-behind period, many people still have the psychological shadow of inferiority and think of others better than themselves.

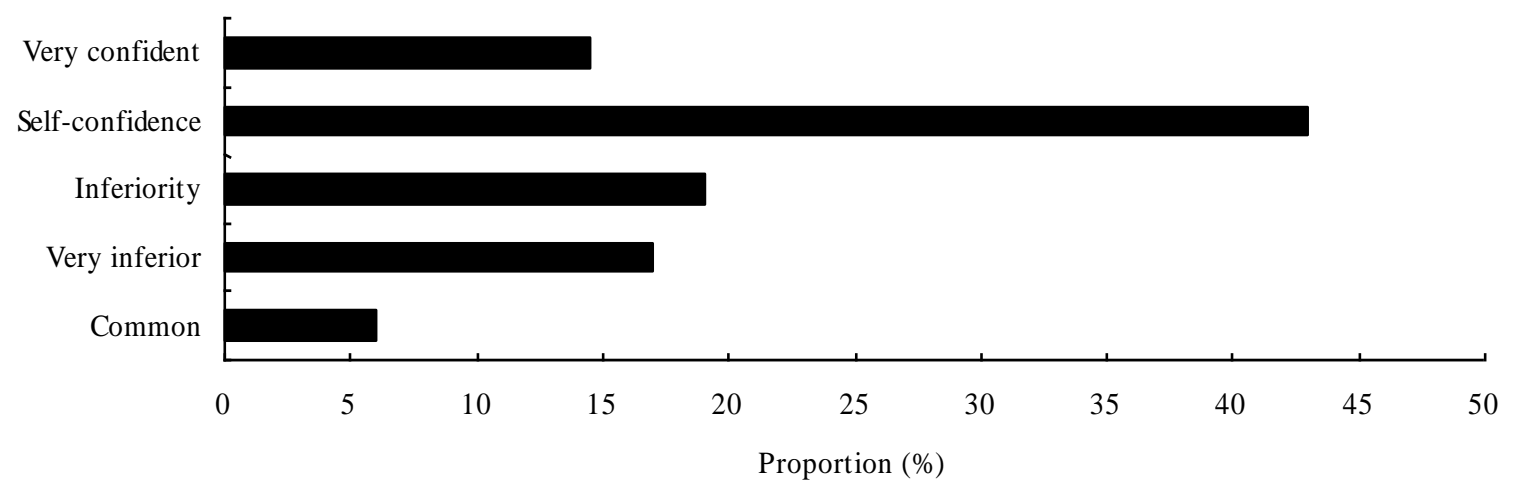

Fig. 4. Degree of confidence

\subsubsection{Temper characteristics}

Many articles have pointed out that the temper of most left-behind children is very bad and the main show is in irascibility, peppery and easy conflicting with others and so on. However, in the questionnaire issued in the study, 58.6\% participants think that their temper is OK, $19.7 \%$ participants think that their temper is very good, only very few think their temper is not so good and no participants choose the item of "A very bad temper". 
The vast majority of college students with left-behind experience have an optimistic attitude to his temper and it seems that their temper and the bad temper of left-behind children are touched at all. However, 54.3\% of 20 other interview students think that the temper of the above college students with left-behind experience is not very good, specific performance in their more sensitive, easy to get angry and often mood fluctuation because of trivialities etc.

\subsubsection{Difference with the surrounding people's personality}

Due to the unique left-behind experience, there is a certain difference between the college students with leftbehind experience and the college students without left-behind experience. For people around the participants, $62.3 \%$ think they are more mature, $85.2 \%$ consider they are more independent and $74.6 \%$ think they are stronger. In fact, because the college students with left-behind experience are loss of parental care too young to be raised by others or take care of themselves during their adolescent period, they are often earlier independent than the people around them. Meanwhile, because parents go out home and enter a city to work, the experience which they have to let them earlier get into the real society and more problems need to be dealt by themselves than the peers make the college students with left-behind experience more mature and stronger than the college students without left-behind experience.

\subsection{Big gender differences in cognition and emotion}

The survey found there are some differences of cognition and emotion between the college boys and girls with left-behind experience.

\subsubsection{Attitude to parents going out to work}

In fact, in order to provide for their family and make a living, although parents were forced willy-nilly to go out to work in the city, the views of the college students with left-behind experience on the matter are not very consistent.

The statistical data in Table 3 showed almost all of the college students with left-behind experience feel that the parents' going out to work is the correct choice. Most of the reasons they gave are for a living, supporting their family, education budget and economic sources. There are still few people consider parents' going out to work to exercise their individual ability and make their way of life wider. In addition, individual students consider parents' going out to work is fashioned and their parents' going out make themselves more freedom. Of course, there are still a few people think that this is not a suitable choice, though the parents' going out lightened the burden of the family and improved economic conditions. They think that children need parental love and the communication between parents and children after the parents' going out. Thus, we can see that most of the college boys with left-behind experience understand their parents' going out to work.

Table 3. University students' views of parents going out to work

\begin{tabular}{lll}
\hline Category & Male university student & Female university student \\
\hline Correct & $93.2 \%$ & $66.7 \%$ \\
Incorrect & $4.5 \%$ & $26.2 \%$ \\
Uncertainty & $2.3 \%$ & $7.1 \%$ \\
\hline
\end{tabular}

However, the views of female university students with left-behind experience on parents' going out to work have a big difference with that of male university students. Only $66.7 \%$ of female university students consider 
that parents' going out to work is the correct choice, obviously, this data is far less than that of male university students. But the proportion which female university students consider parents' going out to work is incorrect reached as high as $26.2 \%$ and the reason is similar to that of male university students.

\subsubsection{Concept of love}

The reasons why college students fall in love differ in thousands of ways. Table 4 shows the reasons why the university students with left-behind experience fall in love.

Data show the love motivation of only $20 \%$ of boys really loves each other, but the love motivation of the girls accounted for 50\%. Most reasons which male university students fall in love are they think it's the suitable time to fall in love. And the love for them is just like having a meal which they should do. So, they fall in love not because of loves, even not owing to willing to fall in love and fall in love. Correspondingly, the other important reason why the female university students fall in love is that they feel fun and want to take a chance.

Table 4. Causes of college students love

\begin{tabular}{llll}
\hline \multicolumn{2}{c}{ Male university students } & & Female university students \\
Category & Proportion $(\%)$ & Category & Proportion $(\%)$ \\
\hline Love & $20 \%$ & Love & $50 \%$ \\
Very boring and leisurely & $8.6 \%$ & Lonely & $4.2 \%$ \\
Suitable time to fall in love & $28.6 \%$ & Amusing and taking a chance & $25 \%$ \\
Psychological need & $11.4 \%$ & Psychological need & $4.2 \%$ \\
Unable to define & $28.5 \%$ & Unable to define & $8.3 \%$ \\
Enrich their university life & $2.9 \%$ & Help each other & $8.3 \%$ \\
\hline
\end{tabular}

In summary, the falling in love motivation of the college students with left-behind experience is very complex. However, the quantity which students fall in love for love is not much, perhaps only accounting for only about $1 / 3$ of the total, and the love motivation of most of the remaining students is not pure. Because of lack of lack of parental care, a lot of left-behind children become lonely and have no sense of security, so they are eager to get care for others to make up the inner emptiness. As a result, they choose to fall in love. It can be seen that these college students' concept of love is sound, especially the male university students.

\subsubsection{Views on university life}

Investigation shows that $12.5 \%$ of the college students with left-behind experience feel that their university life is very happy, $59.4 \%$ feel happier, $18.3 \%$ feel very ordinary and $8.6 \%$ and $1.2 \%$ feel not happy and not very happy respectively. The university life of most college students with left-behind experience is good, even some people feel very happy. Of course, there are a number of people consider their university life is very common, but the proportion is not large.

The author kept count of the views on the university life of college students with left-behind experience (Table 5). In this regard, gender difference is not very noticeable. There are $69.4 \%$ of male university students and $74.2 \%$ of female university students above the general level. Therefore, most students with left-behind experience love their lives very much, and it also reflects their hearts are very energetic. 
Table 5. Views of the college students with left-behind experience on university life

\begin{tabular}{lll}
\hline Category & Male university student & Female university student \\
\hline Loving the university life and full of life & $28.9 \%$ & $22.2 \%$ \\
Okay & $27.5 \%$ & $32.0 \%$ \\
Common & $13.0 \%$ & $20.0 \%$ \\
Boring, empty, meaningless & $26.0 \%$ & $18.0 \%$ \\
Dislike & $4.6 \%$ & $7.8 \%$ \\
\hline
\end{tabular}

\section{Basic factors affecting college students with left-behind experience}

\subsection{Urban-rural binary structure policies and backward rural economy}

With the implementation of urban-rural binary structure policy, urban economy continues to evolve considerably, but the development speed of the rural is very slow. As a result, the gap between urban and rural areas is bigger and bigger. If only relying on a few acres of farmland, it is far from meeting the cost of the home. In order to earn a living of farmers, they had to move to the economically developed city to find more job opportunities.

Simultaneously, because China implemented the household registration system of urban-rural segregation, and according to the regulations produced by the household registration system, children attend school free or low of charge in the site of origin. In this condition, urban migrant workers can hardly bear the high tuition fees. So these students' parents had to leave young children at home [12].

\subsection{Serious family burdens}

According to the statistical results, $39 \%$ of the family population of the guardian is 3; $42 \%$ are 4 (not including grandparents); $17 \%$ are 5 and $2 \%$ are single-parent families. For families without fixed income in rural areas, the more population is, the heavier family will be burdened. And children's tuition fees and the family expenses are a very big number to them.

In order to cope with their children's tuition fees, many college students' parents from the countryside had to drag their no younger body to go out work to earn money. For college students with left-behind experience, there are still a lot of parents working in the city. According to the survey, 52.7\% of parents are still working in nonlocal city. Moreover, the education degree of these parents is low so as to only perform simple manual labor.

\subsection{Influence of parents out during their children's juvenile period}

Farmers working in cities do improve the family economic conditions and provide more possibilities of leftbehind children's education. Instead, a few children become more forceful, more sensible and stronger self-care ability because their parents are not around. But at the same time, parents' going out has a very serious negative effect on the mind education of the left-behind children.

Growth without parental care, left-behind children is like the children who live in a "single-parent families" or "alternate-generation family" $[13,14,15,16]$. In fact, because of the long-term lack of emotion and psychological imbalance, many left-behind children's psychology can't develop nicely. They are usually pessimistic, autism, antagonistic, empty, inferior, timid and no spiritual sustenance and so on.

After the parents went out, they contacted with their children one time even for a long time. The result is the relationship between parents and children are not far less intimate than before. Depending on the survey, $42 \%$ of the college students with left-behind experience consider the relations between them have become some 
strange. Another $17.6 \%$ thinks the relations become very unfamiliar. This makes many children think parents do not care about them, or even blame the parents away from home. In addition, numerous left-behind children have a lot of learning and moral problems because of the lack of parental discipline and the role of a model.

\subsection{Effect of foster experience}

Because husband and wife go out to work in the city, their children have to be brought up by other people. Investigation shows that there is $70.4 \%$ of the college students with left-behind experience are brought up by paternal grandfather, paternal grandmother, maternal grandfather and maternal grandmother. Second, there are $15.7 \%$ people raised by relatives. In addition, the part of self-guardianship reached $13.1 \%$ (Table 6).

The generational guardians are older, low cultural quality and backward thinking. Because of the close kinship, the generational guardians always grant whatever the children's material needs. In addition, owing to the huge age difference, it is easy to produce the generation gap between the guardian and person under guardianship to these left-behind children formed the characters of a withdrawn, introverted and shy [12].

Table 6. Situation of the guardian of the college students with left-behind experience while their parents go out to work

\begin{tabular}{lll}
\hline Category & Total proportion & Proportion \\
\hline Generational guardianship & $70.4 \%$ & $\begin{array}{l}\text { Paternal grandfather and paternal grandmother (46.8\%), Maternal } \\
\text { grandfather and maternal grandmother (20.5\%), Alternation of paternal } \\
\text { grandfather and maternal grandfather (3.1\%) }\end{array}$ \\
Relative guardianship & $15.7 \%$ & $\begin{array}{l}\text { Father's elder brother (7\%), Father's younger brother (3\%), Father's sister } \\
(1.6 \%), \text { mother's brother (0.8\%), Aunt }(2.5 \%)\end{array}$ \\
Self guardianship & $13.1 \%$ & Sister and brother (3.3\%), oneself (9.8\%) \\
Neighbor guardianship & $0.8 \%$ & Neighbor $(0.8 \%)$ \\
\hline
\end{tabular}

\section{Conclusion}

College students with left-behind experience have their distinctive characteristics. For the economy, their family income is generally low, and some of them are even very difficult to maintain the basic needs of their life. On the study, although most of these students' learning attitudes are OK, and the class enthusiasm and serious degree are also relatively high, their academic performances are still below the average levels. For the interpersonal relationship, the college students with left-behind experience generally don't like social activities, and their interpersonal communication abilities are also poor and some of them even have severe communication difficulties. Their personality tendency is as with ordinary people, and it seems to be more mature, strong and independent. However, quite a few people still look very inferior, and there are less than 1/3 of the people in a good temper. For emotional cognition, a certain percentage of people's values, philosophy and emotional views are more extreme and not perfect. In short, it can still be seen that the remaining problems of left-behind children from the college students with left-behind experience, even a part of the performance were very prominent.

In author's investigation, it found that there the quantity of the college students with left-behind experience is a lot in the whole college students. And with the popularity of "the tide of migrant workers", this proportion will be greater and greater. Their quality will relate to the social development and the future direction closely. Therefore, improving the living situation of the college students with left-behind experience is an urgent problem to be resolved. However, seeking both temporary and permanent solutions while focusing on the permanent solution, the key to solving this problem lies in solving the problem of left-behind child.

The generation of left-behind children is a necessary phenomenon during the Chinese social transformation period with the profound background of the traditional urban-rural opposition thinking mode. In the face of the fact that the rural population accounts for more than half Chinese, the problem of left-behind children has 
already become a serious social problem. Left-behind children problem solving will relate to the development of rural areas, the urban and rural construction, the quality and cultivation of college students and the future construction and development of China. So, we should solve these problems jointly by practical understanding left-behind children's situation, strengthening the left-behind children's problems advocacy, mobilizing the government, schools, parents and people from every part of society.

\section{Acknowledgements}

Financial support from teaching reform project of "The research and practice of cooperative education mode of geographical science" (G21110, supported by Hunan Provincial Education Department); Project of Science and Technology Department of Hunan Province (2013RS4053); Social Science Fund of Hunan Province (12YBA136).

\section{References}

[1] Duan Chengrong, Zhou Fulin. A study on children left behind. Population Research, 2005; 29 (1):29-36.

[2] Wang Fan. Analysis on social security problems of migrant workers during the Urbanisation process in China. Hubei Social Sciences, 2008, 3: 66-68.

[3] Shang Yuxin, Zhu Honghui. Reason and condition analysis on rural and urban mobility of migrant workers. Rural Economy and Science-Technology, 2010, 3: 33-35.

[4] Chi Xixin. An analysis on the moral development problems of unattended children from a psychosocial perspective. Teacher Education Research, 2005,17 (6):72-75.

[5] Wang Hongmei. Survey and Thinking on Entrepreneurship education of Local College Students. I.J. Education and Management Engineering, 2012, 8, 25-30

[6] Lina Yu. Research On the "Cooperative Education" Model Cultivating In Higher Vocational Education. I.J. Education and Management Engineering, 2012, 1,35-41

[7] Zhiyan JIA, Xie Han. Construction of Evaluation System on Multimedia Educational Software. I.J. Education and Management Engineering, 2013, 1, 34-38

[8] Zhang Wenhua, Kong Ping. Intervention study on the team work in left-behind children protection. Youth \& Juvenile Research, 2011,3:27-30.

[9] Wang Hui. The psychological problems of left-behind children in rural areas. Money China, 2013, 2: 104106.

[10] Wang Jingfeng. Psychology and education problems and Countermeasures of rural left-behind children. Continue Education Research, 2010, 4: 71-73.

[11] Wang Jingfeng. Psychological and Educational Issues and Causes of Left-behind Children in Rural Areas. 2010, 30 (2): 141-143.

[12] Li Chenxu. An education problem of "Rural left-behind children" should be solved quickly. Guang Ming Daily, 2002, 4, 9.

[13] Li Qingfeng. Series of Children's data interpretation (Fourth Series): Development status of left-behind children. 2005, 3, 8 (http://www.cycs.org/Article.asp?ID=4181).

[14] Cao Guangzhong, Bian Xue, Zhao Jinhua. The Structural Character and Spatial Disparity of Left-behind Families in rural China: Evidence from a Nationwide Survey in 30 Counties. Population and Development, 2013,19(4):2-10.

[15] Ma Fang. Guardian's impact on the psychological health of left-behind children. Journal of Anhui Agricultural Sciences, 2008, 36 (10): 4293-4294, 4298.

[16] Li Cuiying. Parent-child model of left-behind children in rural areas. Journal of Hunan University of Science and Engineering, 2009, 30 (9): 9-10. 


\section{Author(s) Profile}

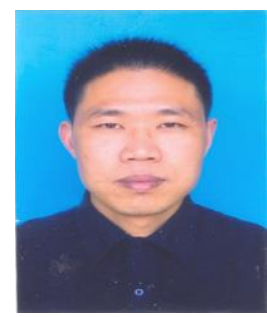

Zhang Yong, $\mathrm{PhD}$ is a professor at the Department of Geography, School of Architecture and Urban Planning of Hunan University of Science and Technology. His research interests concern the teaching of Higher Education, the Geography teaching, evolution of global change and the environment.

Postal address: School of Architecture and Urban Planning of Hunan University of Science and Technology, Xiangtan, 41120, China.

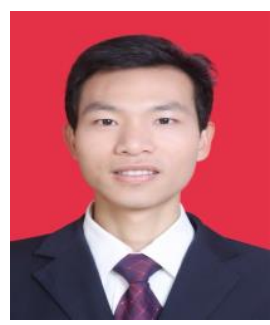

Jiang Wulin, Graduate of Hunan University of Science and Technology, School of Architecture and Urban Planning of Hunan University of Science and Technology. His research interests concern the teaching of Higher Education, the Geography teaching, environmental pollution.

Postal address: School of Architecture and Urban Planning of Hunan University of Science and Technology, Xiangtan, 41120, China.

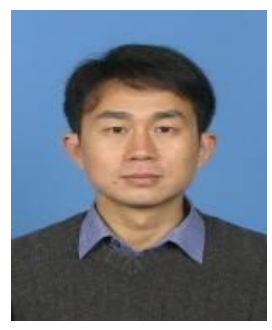

Xiang Yunbo, $\mathrm{PhD}$ is a lecturer at the Department of Geography, School of Architecture and Urban Planning of Hunan University of Science and Technology. His research interests concern the teaching of Higher Education, the Geography teaching, tourism geography, tourism planning, human geography.

Postal address: School of Architecture and Urban Planning of Hunan University of Science and Technology, Xiangtan, 41120, China.

How to cite this paper: Zhang Yong, Jiang Wulina, Xiang Yunbo,"Investigation and Study on the Status of the College Students with Left-Behind Experience in China", IJEME, vol.4, no.2, pp.18-28, 2014.DOI: 10.5815/ijeme.2014.02.03 